\title{
Finite Element Implementation of a Peridynamic Pitting \\ Corrosion Damage Model
}

\author{
Dennj De Meo*, Erkan Oterkus** \\ * Department of Naval Architecture, Ocean and Marine Engineering University of \\ Strathclyde \\ 100 Montrose Street Glasgow G4 0LZ, United Kingdom \\ Phone: $+44(0) 1415484094$ \\ e-mail: dennj.demeo@strath.ac.uk \\ ** Department of Naval Architecture, Ocean and Marine Engineering University of \\ Strathclyde \\ 100 Montrose Street Glasgow G4 0LZ, United Kingdom \\ Phone: $+44(0) 1415483876$ \\ e-mail: erkan.oterkus@strath.ac.uk
}

\begin{abstract}
Despite the significant improvements in the understanding of pitting corrosion, many aspects of this phenomenon remain unclear and corrosion rate prediction based on experimental data remains difficult. Experimental measurements of corrosion rates under different electrochemical conditions can be complex and time consuming, and the conclusions are limited to the timescale and the conditions in which experiments have been carried out. In order to overcome these limitations, numerical approaches can be a valuable complement. Hence, in this study a new numerical model based on peridynamics to predict pitting corrosion damage is developed. The developed model is implemented in a commercial finite element software and it allows for the reproduction of realistic pitting morphologies, modelling of microstructural effects such as the presence of intermetallic particles and the reduction of the computational cost of the simulations. In conclusion, the results of this study shows that the peridynamic models can be helpful in failure analysis and design of new corrosion-resistant materials.
\end{abstract}


Keywords: Pitting; Corrosion; Peridynamics; Damage; Finite Element Method

\section{Introduction}

Corrosion is a natural process that can be described as the chemical or electrochemical reaction of an engineering material with the environment, which eventually leads to the deterioration of its mechanical properties. There are various types of corrosion mechanisms including stress corrosion cracking (SCC) and pitting corrosion.

Pitting is a localised form of corrosion that leads to the formation of corrosion cavities or pits due to the breakage of the material's passive film. Pitting typically occurs in materials such as stainless steel, aluminium, titanium, copper, magnesium and nickel alloys. Pitting corrosion is the most common, dangerous and destructive type of corrosion in marine and offshore structures (Bhandari et al., 2015).

Despite the significant improvements in the understanding of pitting corrosion, many aspects of this phenomenon remain unclear and corrosion rate prediction based on experimental data remains difficult (Onishi et al., 2012). Experimental measurements of corrosion rates under different electrochemical conditions can be complex and time consuming, and the conclusions are limited to the timescale and the conditions in which experiments have been carried out (Van der Weeën et al., 2014). Moreover, distinguishing the effect of each individual variable becomes extremely difficult (Xiao and Chaudhuri, 2011). In order to overcome these limitations, numerical approaches can be a valuable complement.

Several numerical approaches are available in the literature for the modelling of corrosion damage, and many of them are based on finite element method (Sharland et al., 1989; Walton, 1990; Laycock and White, 2001; Scheiner and Hellmich, 2009). However, the modelling of pit propagation by using finite element method (FEM) is not an easy task since special procedures of moving mesh and remeshing are often needed (Duddu, 2014). Moreover, if remeshing techniques are used, the finite element matrices have to be recalculated for every time step (Vagbharathi and Gopalakrishnan, 2014). In order to overcome these difficulties, an alternative 
numerical approach to the FEM can be the use of extended Finite Element Method (XFEM). Vagbharathi and Gopalakrishnan (2014) and Duddu (2014) describe numerical models of corrosion pit propagation in stainless steel based on the XFEM framework and Fick's law of diffusion. An extension to the latter work was done by Duddu et al. (2015), where the Fick's law of diffusion was replaced by the NernstPlanck equation and crevice corrosion in $\mathrm{Al}-\mathrm{Mg}$ alloy microstructures was investigated.

Although all the aforementioned models have been quite effective in predicting the corrosion rates reported in the experimental literature, none of these models have been able to predict corrosion subsurface damage, which is a phenomenon documented in the experimental literature (Song et al., 2014). With the aim of filling this gap, a new continuum mechanics formulation, peridynamics (PD), has been recently used to create a model of pitting corrosion (Chen and Bobaru, 2015) based on a modified Nernst-Planck equation. This work was extended in (Chen et al,, 2015), where the influence of the pit cover on the pit morphology was investigated.

In this study, a new numerical model based on PD theory for the investigation of pitting corrosion is introduced. This work is based on the PD model of pitting corrosion developed by Chen and Bobaru (2015). However, the numerical approach used in the present study is different and offers the advantage of a reduced computational time by performing the numerical implementation in a commercial finite element software and utilizing implicit time integration. Moreover, the capabilities of the numerical framework have been extended to the modelling of realistic pit morphologies and determining the influence of microstructural features of the material, e.g. intermetallic particles. The ultimate goal of this study is to produce a non-conventional and more effective numerical framework that can be helpful in failure analysis and design of new corrosion-resistant materials. 


\section{Peridynamics}

The governing equations of classical continuum mechanics (CCM) are based on partial differential equations (PDEs) and its mathematical formulation breaks down in the presence of discontinuities such as cracks. This limitation is partially overcome with the adoption of external crack growth criteria based on fracture mechanics. However, this approach presents its own limitations. In light of the limiting assumptions and difficulties of the current approaches, a new mathematical formulation of continuum mechanics was developed by Silling (2000), which is called "peridynamics".

The governing equations of PD are integro-differential. The PD equations of motion of a generic material point $\mathbf{x}$ can be written as (Silling, 2000)

$$
\rho(\mathbf{x}) \ddot{\mathbf{u}}(\mathbf{x}, t)=\int_{H_{\mathbf{x}}} \mathbf{f}\left(\mathbf{u}\left(\mathbf{x}^{\prime}, t\right)-\mathbf{u}(\mathbf{x}, t), \mathbf{x}^{\prime}-\mathbf{x}\right) \mathrm{d} V_{\mathbf{x}^{\prime}}+\mathbf{b}(\mathbf{x}, t)
$$

where $\rho(\mathbf{x})$ and $\ddot{\mathbf{u}}(\mathbf{x}, t)$ denote the density and acceleration of the material point $\mathbf{x}$ at time $t$, respectively. In Eq. (1), $\mathbf{f}\left(\mathbf{u}\left(\mathbf{x}^{\prime}, t\right)-\mathbf{u}(\mathbf{x}, t), \mathbf{x}^{\prime}-\mathbf{x}\right)$ represents the PD force between material points $\mathbf{x}$ and $\mathbf{x}^{\prime}$ (also called mechanical response function) and the term $\mathbf{b}(\mathbf{x}, t)$ is the body force acting on material point $\mathbf{x}$ at time $t . \mathrm{d} V_{\mathbf{x}^{\prime}}$ is the volume associated with material point $\mathbf{x}^{\prime}$. According to this new formulation, a material point can interact with other material points not only within its nearest neighbourhood, but also with material points in a larger neighbourhood (Fig. 1). 


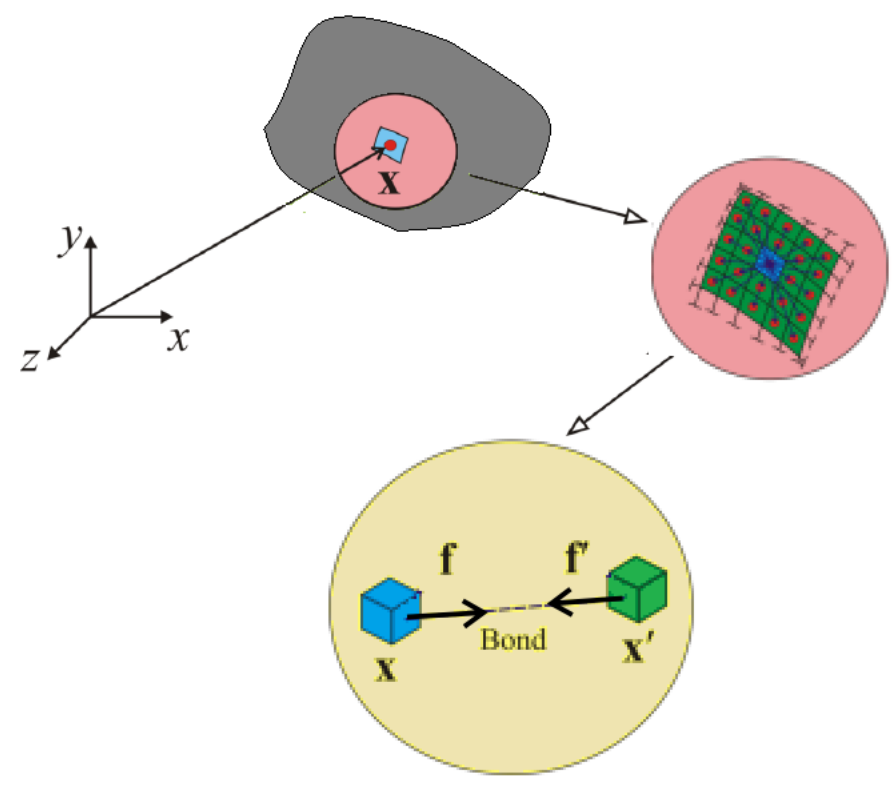

Fig. 1 Peridynamic forces between between material points $\mathbf{x}$ and $\mathbf{x}^{\prime}$.

It can be assumed that the interaction between material points decreases as the distance between them increases. Therefore, an influence domain, named horizon, $H_{\mathrm{x}}$, can be defined for each material point as shown in Fig. 2.

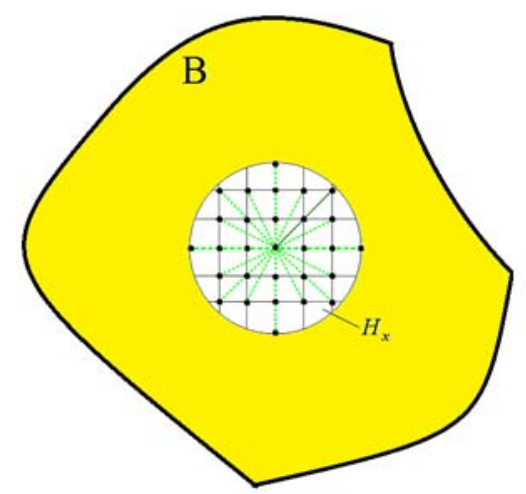

Fig. 2 Peridynamic bonds between material points inside the peridynamic horizon, $H_{\mathbf{x}}$.

The material point $\mathbf{x}$ can only interact with material points within this domain, which are called the "family" of $\mathbf{x}$. This interaction is called "bond" and its length is simply the distance between the two material points. In the case of an elastic 
material, the peridynamic force between material points $\mathbf{x}$ and $\mathbf{x}^{\prime}$, can be expressed as

$$
\mathbf{f}=\operatorname{cs} \frac{\mathbf{y}^{\prime}-\mathbf{y}}{\left|\mathbf{y}^{\prime}-\mathbf{y}\right|}
$$

where $\mathbf{y}$ represents the location of the material point $\mathbf{x}$ in the deformed configuration as shown in Fig. 3, i.e. $\mathbf{y}=\mathbf{x}+\mathbf{u}$, while $c$ is the bond constant which can be related to material constants of CCM as described in Madenci and Oterkus (2014).

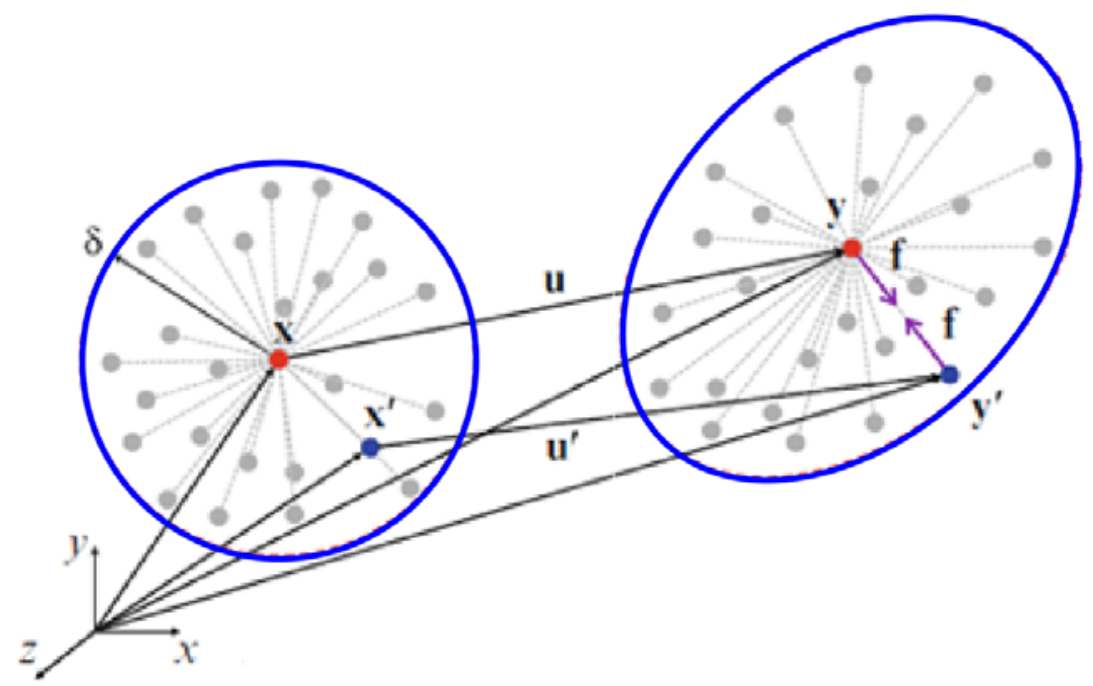

Fig. 3 Peridynamic horizon in the undeformed configuration (left) and the deformed configuration (right)

In Eq. (2), the stretch parameter, $s$, is defined as

$$
s=\frac{\left|\mathbf{y}^{\prime}-\mathbf{y}\right|-\left|\mathbf{x}^{\prime}-\mathbf{x}\right|}{\left|\mathbf{x}^{\prime}-\mathbf{x}\right|}
$$

In the case of a brittle material behaviour, the peridynamic force and the stretch relationship are shown in Fig. 4. 


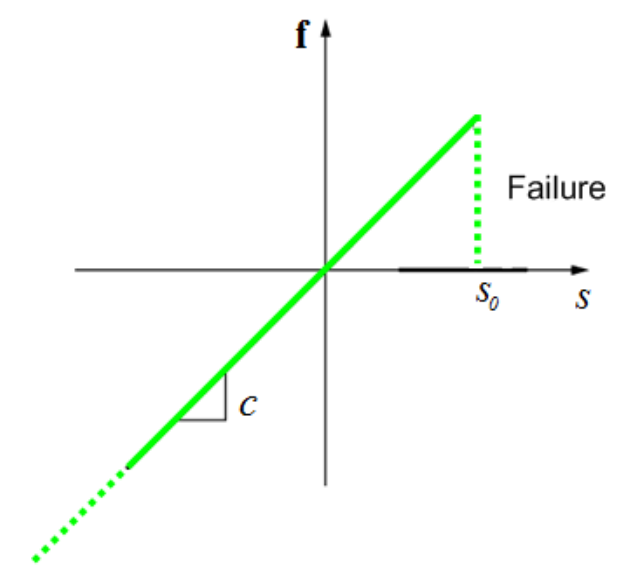

Fig. 4 PD bond behaviour for brittle materials

The parameter $s_{0}$, in Fig. 4, is called critical stretch and if the stretch of a peridynamic bond exceeds this critical value, the peridynamic interaction (bond) is broken. As a result, the peridynamic force between the two material points reduces to zero and the load is redistributed among the other bonds, leading to unguided material failure. Peridynamics can be applicable for different material systems including metals and composites (Oterkus and Madenci, 2012). Moreover, PD framework can be extended to other fields such as thermal (Gerstle et al., 2008), moisture (Oterkus et al., 2014), etc., so that it can be used as a single platform for multiphysics analysis of materials (Amani et al., 2016; De Meo et al., 2016; Oterkus et al., 2013; Oterkus and Madenci, 2014).

\section{Peridynamic modelling of pitting corrosion}

In this section, PD framework for modelling of pitting corrosion based on the formulation developed by Chen and Bobaru (2015) is presented. The mathematical formulation is based on a modified version of the Nernst-Planck equation (NPE). The NPE is a mass conservation equation that describes the motion of charged chemical species in a fluid under the effect of concentration gradients (diffusion), electric field (migration) and fluid velocity (convection). In this regard, the flux $\mathbf{N}_{i}$ in $\left[\mathrm{mol} /\left(\mathrm{m}^{2} / \mathrm{s}\right)\right]$ of the generic species ' $i$ ' can be written as (Gavrilov et al., 2007; Chen and Bobaru, 2015) 


$$
\mathbf{N}_{i}=\underbrace{-D_{i} \nabla C_{i}}_{\text {diffusion }}-\underbrace{\frac{\mathrm{F}}{\mathrm{R} T} n_{i} D_{i} C_{i} \nabla \phi}_{\text {electro-migration }}+\underbrace{C_{i} \mathbf{U}}_{\text {convection }}
$$

where $D_{i}$ in $\left[\mathrm{m}^{2} / \mathrm{s}\right]$ is the diffusion coefficient, $C_{i}$ is the concentration in $\left[\mathrm{mol} / \mathrm{m}^{3}\right]$ of the species " $i$ ", $\mathrm{F}$ in $[\mathrm{C} / \mathrm{mol}]$ is the Faraday constant, $\mathrm{R}$ in $[\mathrm{J} /(\mathrm{mol} \mathrm{K})]$ is the universal gas constant, $n_{i}$ is the valence number, $\phi$ in $[\mathrm{V}]$ or $[\mathrm{J} / \mathrm{C}]$ is the electric potential and $\mathbf{U}$ in $[\mathrm{m} / \mathrm{s}]$ is the flow velocity. The conservation of mass can be written as (Gavrilov et al., 2007)

$$
\frac{\partial C_{i}}{\partial t}=-\nabla \cdot \mathbf{N}_{i}
$$

where $t$ in [s] is time and $\nabla \cdot$ is the divergence operator. The majority of pitting corrosion models available in the literature focuses on the motion of chemical species inside the electrolyte solution. However, recent experimental studies have revealed the existence of a 'wet region' at the solid/liquid interface of the corrosion pit, where the motion of chemical species can occur. In other words, the electrolyte solution penetrates the subsurface region of the pit filling the pores of the material, allowing metal dissolution to occur not only at the solid/liquid interface, but also in a subsurface region of the pit.

In order to capture this process, Eq. (5) is used to predict the motion of metal cations $\mathrm{Me}^{n+}$ in both the electrolyte solution and the solid with the following simplifications and assumptions:

\section{Motion within the solution}

- The electromigration and convective terms in Eq. (4) are included inside the diffusion coefficient, which is now called as effective diffusion coefficient in the liquid $D_{l d}$. 
Therefore, the governing equation used to predict the motion of metal cations within the electrolyte solution is given by the following modified version of the NernstPlanck equation as

$$
\frac{\partial C_{M I}}{\partial t}=-\nabla \cdot\left(-D_{l d} \nabla C_{M I}\right)=D_{l d} \nabla^{2} C_{M I}
$$

where, $C_{M I}$ refers to the metal concentration inside the electrolyte solution, which is considered to be $1 \mathrm{M} \mathrm{NaCl}$ in this study. The effective diffusion coefficient in the liquid can be obtained from the study reported in Kuo and Landolt (1975), where rotating disk electrode experiments of iron dissolution in chloride solutions are described and the electromigration effect is taken into consideration empirically as

$$
D_{l d}=8.5 \cdot 10^{-10} \mathrm{~m}^{2} / \mathrm{s}
$$

\section{$\underline{\text { Motion within the solid }}$}

- The velocity of the fluid diffusing inside the pores of the metal is neglected and the convection term in Eq. (4) is not considered.

- The effect of the electromigration term in Eq. (4) is included inside the diffusion coefficient, which is now called as effective diffusion coefficient in the solid $D_{s d}$.

The governing equation used to describe the motion of metal cations within the solid is given by the following modified version of the Nernst-Planck equation as

$$
\frac{\partial C_{M I}}{\partial t}=-\nabla \cdot\left(-D_{s d} \nabla C_{M I}\right)=D_{s d} \nabla^{2} C_{M I}
$$

where, in this case, $C_{M I}$ refers to the metal concentration inside the solid.

As shown in Eq. (8), the flux of metal cations is linearly proportional to the effective diffusivity. According to Faraday's laws of electrolysis, the amount of 
metal dissolution $\mathbf{N}_{a}$ in $[\mathrm{mol} / \mathrm{s}]$ is linearly proportional to the anodic current density, which can be expressed as

$$
i_{a}=n \mathrm{~F} \mathbf{N}_{a}=n \mathrm{~F} \frac{d M_{o l}}{d t}
$$

where $M_{o l}$ expressed in [mol] is the number of moles of the material. Therefore, in order to obey Faraday's laws of electrolysis, the effective diffusion coefficient in the solid is expressed as a function of the overpotential $\eta$ as

$$
D_{s d}=D_{s d}(\eta)=D_{s d}(0) e^{\frac{\alpha n \mathrm{~F} \eta}{\mathrm{R} T}}
$$

In Eq. (10), the term $D_{s d}(0)$ represents the value of the diffusion coefficient in the solid when the overpotential is null, which can be found through calibration against experimental polarisation data. Eq. (10) can also be written as

$$
\eta=\beta_{a} \cdot \log \left(\frac{D_{s d}(\eta)}{D_{s d}(0)}\right)
$$

Moreover, the anodic current density in Eq. (9) can also be expressed as a function of the moving speed of the solid/liquid interface, i.e. the pit base, $v_{\text {int }}[\mathrm{m} / \mathrm{s}]$ as

$$
i_{a}=n \mathrm{FN}_{a}=n \mathrm{~F} C_{\text {solid }} v_{\mathrm{int}}
$$

where $\mathrm{F}=9.64859 \cdot 10^{4} \mathrm{C} / \mathrm{mol}$ and $C_{\text {solid }}$ in $\left[\mathrm{mol} / \mathrm{m}^{3}\right]$ is the concentration of metal cations in the solid. As described in Scheiner and Hellmich (2009), in the case of stainless steel grade 304 (304 SS), i.e. the material considered in this study, $C_{\text {solid }}$ can be calculated by considering the mass density of 304 SS and the molar masses of its main components $\mathrm{Fe}, \mathrm{Ni}$ and $\mathrm{Cr}$ as

$$
C_{\text {solid }}=143,000 \mathrm{~mol} / \mathrm{m}^{3}
$$

A similar approach can be used for the calculation of the valence number of $304 \mathrm{SS}$ : 


$$
n=2.19
$$

As mentioned earlier, the corrosive solution considered in this study is $1 \mathrm{M} \mathrm{NaCl}$ at temperature $T=298.15 \mathrm{~K}$ and the related saturation concentration value of dissolved stainless steel metal cations, $C_{s a t}$, can be inferred from X-ray analysis (Isaacs et al., 1995) as

$$
C_{\text {sat }}=5,100 \mathrm{~mol} / \mathrm{m}^{3}
$$

As described in Chen and Bobaru (2015), the calibration procedure for the calculation of $D_{s d}(0)$ consists of the following steps:

1. A small initial value is chosen for the diffusion coefficient in the solid $D_{s d}(\eta)$ to make sure that corrosion will be in activation-controlled regime.

2. The simulation is launched and the velocity $v_{\text {int }}$ of the solid/liquid interface, i.e. metal dissolution front, is recorded.

3. Eq. (12) is used to calculate the current density, $i_{a}$.

4. Once $i_{a}$ is known, it is possible to calculate the corresponding overpotential value by using the experimental polarization diagram reported in Chen and Bobaru (2015).

5. Eq. (11) can now be used to calculate $D_{s d}(0)$ considering the following value of the Tafel constant: $\beta_{a}=45.1 \mathrm{mV}$ (Chen and Bobaru, 2015).

$D_{s d}(0)=7.352 \cdot 10^{-17} \mathrm{~m}^{2} / \mathrm{s}$ is the outcome of this calibration procedure, which is in agreement with Chen and Bobaru (2015).

When the concentration of metal ions in the liquid reaches the saturation value $C_{s a t}$, a salt film precipitates at the liquid/solid interface. The concentration of metal 
ions in the liquid cannot be greater than the saturation value. When the concentration value of the generic node is greater than $C_{s a t}$, then the node is considered to be in solid phase. On the contrary, when the concentration value is smaller than $C_{\text {sat }}$, then the node is considered to be in liquid phase.

The peridynamic governing equation for metal dissolution can be written as

$$
\dot{C}_{M I}(\mathbf{x}, t)=\int_{H_{\mathbf{x}}} f_{\mathrm{d}}\left(C_{M I}(\mathbf{x}, t), C_{M I}\left(\mathbf{x}^{\prime}, t\right), \mathbf{x}^{\prime}, \mathbf{x}, t\right) \mathrm{d} V_{\mathbf{x}^{\prime}}
$$

where $\dot{C}_{M I}(\mathbf{x}, t)$ is the time derivative of metal ions concentration associated with the generic material point $\mathbf{x}$. In Eq. (16), the peridynamic function $f_{\mathrm{d}}\left(C_{M I}(\mathbf{x}, t), C_{M I}\left(\mathbf{x}^{\prime}, t\right), \mathbf{x}^{\prime}, \mathbf{x}, t\right)$ is called metal dissolution response function and it is defined as

$$
f_{\mathrm{d}}=d_{M I} \frac{C_{M I}\left(\mathbf{x}^{\prime}, t\right)-C_{M I}(\mathbf{x}, t)}{\left|\mathbf{x}^{\prime}-\mathbf{x}\right|}
$$

in which the peridynamic metal ions diffusion bond constant $d_{M I}$ can be expressed in terms of the effective diffusion coefficient as (Chen and Bobaru, 2015)

$$
\text { (2D) } \quad d_{M I}=\frac{4 \cdot D_{e f f}}{\pi \cdot \delta^{2} \cdot h}
$$

where $h$ in $[\mathrm{m}]$ refers to the thickness of the body and $D_{\text {eff }}$ in $\left[\mathrm{m}^{2} / \mathrm{s}\right]$ is the effective diffusion coefficient which can be calculated as suggested in Chen and Bobaru (2015) as 


$$
D_{e f f}= \begin{cases}D_{s d} & \text { if } C_{i}>C_{s a t} \text { and } C_{j}>C_{s a t} \\ D_{l d} & \text { if } C_{i}<C_{s a t} \text { and } C_{j}<C_{s a t} \\ \frac{2 \cdot D_{s d} \cdot D_{l d}}{D_{s d}+D_{l d}} & \text { if } C_{i} \leq C_{s a t} \text { and } C_{j} \geq C_{s a t} \\ \frac{2 \cdot D_{s d} \cdot D_{l d}}{D_{s d}+D_{l d}} & \text { if } C_{i} \geq C_{s a t} \text { and } C_{j} \leq C_{s a t}\end{cases}
$$

The PD model of pitting corrosion consists of mechanical bonds overlapped by diffusion bonds. The former bonds are aimed to capture the subsurface mechanical damage reported in recent experimental studies (Song at al. 2014). For this purpose, a damage index, $d(\mathbf{x}, t)$, is defined for each node as

$$
d(\mathbf{x}, t)=\frac{N_{f}}{N_{t o t}}
$$

where $N_{f}$ and $N_{\text {tot }}$ are the number of failed bonds and the total number of bonds attached to the node at $\mathbf{x}$, respectively. At the beginning of the simulation, all the nodes belonging to the solid body have a concentration value $C_{M I}(\mathbf{x}, t)$ equal to $C_{\text {solid }}$, which means no corrosion. Therefore, in this condition, all the mechanical bonds connected to $\mathbf{x}$ are intact and its damage $d(\mathbf{x}, t)$ index is null. On the contrary, when $C_{M I}(\mathbf{x}, t)$ becomes smaller than $C_{\text {liquid }}$, the node at $\mathbf{x}$ changes its phase from solid to liquid, which means complete metal dissolution. Therefore, in this condition, all the mechanical bonds connected to $\mathbf{x}$ are broken and the damage index has a unit value. As suggested in Chen and Bobaru (2015), the damage index $d(\mathbf{x}, t)$ can therefore be written as a function of the nodal concentration $C_{M I}(\mathbf{x}, t)$ as

$$
d(\mathbf{x}, t)=\frac{C_{\text {solid }}-C_{M I}(\mathbf{x}, t)}{C_{\text {solid }}-C_{\text {sat }}}
$$

Each bond has a certain probability $P_{r}$ to be broken in the current time step. In each time step, $P_{r}$ can be calculated as (Chen and Bobaru, 2015) 


$$
P_{r}=\frac{1}{1-d_{t-1}} \cdot\left(\frac{\Delta C_{i}}{C_{\text {solid }}-C_{\text {sat }}}\right)
$$

where $d_{t-1}$ represents the value of the damage index at the previous time step, and $\Delta C_{i}$ is the difference in nodal concentration between the previous time step and the current time step. Once $P_{r}$ is calculated, a random number in the range $[0,1]$ is generated for each bond. If the random number is smaller than $P_{r}$, then the mechanical bond is broken and the value of the damage index is updated.

\section{Numerical solution method}

As mentioned earlier, the PD equation of motion is integro-differential and, in general, it cannot be solved analytically. Therefore, numerical techniques for time and spatial integration are usually needed to solve the PD governing equations.

Concerning spatial integration, a meshless scheme and the collocation method can be used. The domain can be divided in smaller parts (Fig. 5), where each part has an associated volume and integration material point, which is also called as collocation point. In the case of a uniform grid, the distance among the material points is same in all directions.
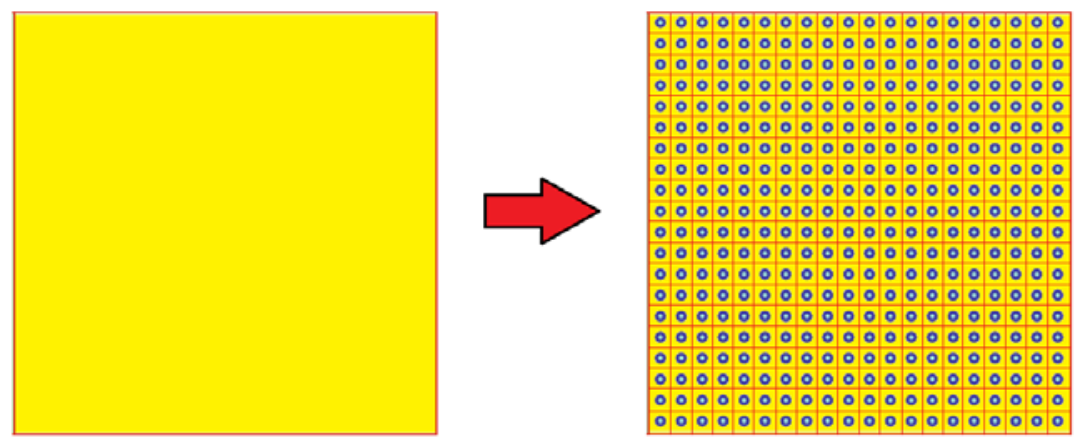

Fig. 5 Discretisation of PD domain in 2-Dimensional configurations.

For a generic material point $\mathbf{x}$, the spatial integration is performed only over the part of the body that is contained within the horizon of the particle $\mathbf{x}$. Therefore, for a generic PD particle $\mathbf{x}_{i}$, the discretised form of Eq. (1) can be written as 


$$
\dot{C}_{M I}(\mathbf{x}, t)=\sum_{j}^{M} f_{\mathrm{d}}\left(C_{M I}\left(\mathbf{x}_{j}, t\right)-C_{M I}\left(\mathbf{x}_{i}, t\right), \mathbf{x}_{j}-\mathbf{x}_{i}\right) V_{j}
$$

where $M$ is the number of family members of particle $\mathbf{x}_{i}$. Concerning the time integration, implicit time integration scheme has been utilized in this study. Eq. (23) can be solved by using either explicit or implicit time integration schemes. The advantage of an implicit time integration scheme is the numerical stability of the method regardless of the value of the time step size. This is particularly important when modelling corrosion, since the timescale of the problem can range from seconds to years. In this study, a novel approach, based on the analogy between heat transfer and metal ions diffusion, is used to exploit the implicit solver implemented in the commercial finite element analysis software ANSYS 14.5.

Thus, the PD model of pitting corrosion can be represented by the following thermal finite elements available in ANSYS:

- MASS 71: Thermal mass elements are used to represent the PD particles. The only degree of freedom is the temperature, which, in our model, corresponds to the metal ions concentration $\left[\mathrm{mol} / \mathrm{m}^{3}\right]$.

- COMBIN 14: Thermal spring elements are utilized to represent the PD bonds. These elements are used to connect the thermal mass elements to each other. As for the MASS 71 elements, the only degree of freedom is the temperature.

The expression for the real constant $d_{A N S Y S}$ of the generic COMBIN 14 element can be written as

(2D) $\quad d_{A N S Y S}=\frac{d_{M I} \cdot s_{c f} \cdot V \cdot v_{c} \cdot V}{l^{2}}$ 
where $l$ in $[\mathrm{m}]$ represents the length of the bond and $V$ in $\left[\mathrm{m}^{3}\right]$ represents the volume a material point. In Eq. (24), $s_{c f}$ and $v_{c}$ are surface and volume corrections, respectively, which are explained in Madenci and Oterkus (2014).

\section{Numerical results}

In this section, three different cases are presented. In the first case, pitting corrosion evolution in a stainless steel plate is simulated for validation purposes. In the second case, more realistic pit morphologies are generated. Finally, the effect of intermetallic particles on pitting corrosion evolution is investigated.

\subsection{Pitting corrosion in a stainless steel plate}

In order to validate the current approach, a 2D plate of dimensions $0.1 \mathrm{~mm} \times 0.1$ $\mathrm{mm}$ and thickness of $1 \mu \mathrm{m}$ is considered. As shown in Fig. 6, the top edge of the plate is damaged and the initial pit is filled with $1 \mathrm{M} \mathrm{NaCl}$ aqueous solution, in which the concentration of metal ions is initially null. The volume occupied by the solution is assumed to be much larger than that of the metal, i.e. austenitic stainless steel grade 304. This assumption allows to represent the bulk of the corrosive solution with a zero-concentration boundary condition throughout the simulation.

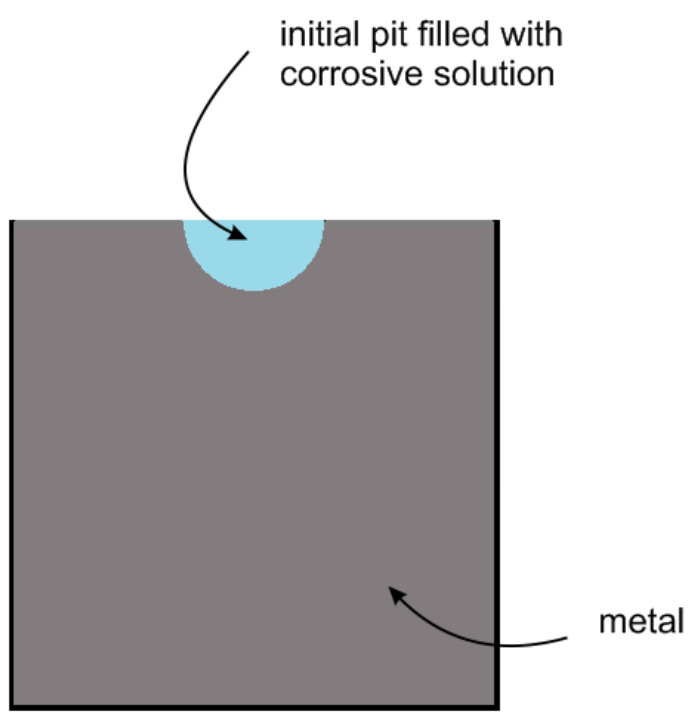

Fig. 6 2D pitting corrosion model: initial pit (blue colour) and metal (grey colour) 
The material data can be found in Section 3. The radius of the initial pit is $10 \mu \mathrm{m}$. The plate is discretised with $100 \times 100$ PD nodes and the resulting value of grid spacing and horizon's radius are $\Delta=1 \mu \mathrm{m}$ and $\delta=4 \mu \mathrm{m}$, respectively. At the beginning of the simulation, all the nodes belonging to the metal have a concentration of metal ions equal to $C_{\text {solid }}$. The appropriate time step size is found by performing a convergence analysis. As shown in Fig. 7, a time step size of $0.5 \mathrm{~s}$ can be considered as a suitable value. The results obtained by using the present model are in qualitative agreement with the numerical results presented in Chen and Bobaru (2015), where a time step size of $0.05 \mathrm{~s}$ is used (see Fig. 8).

A

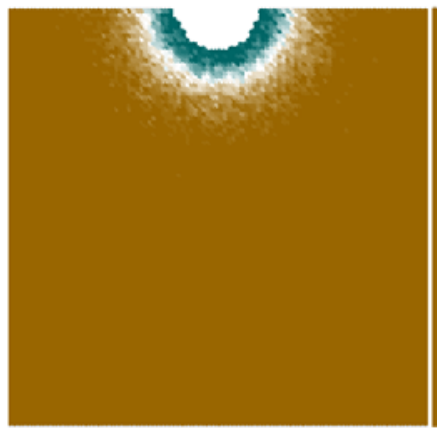

B

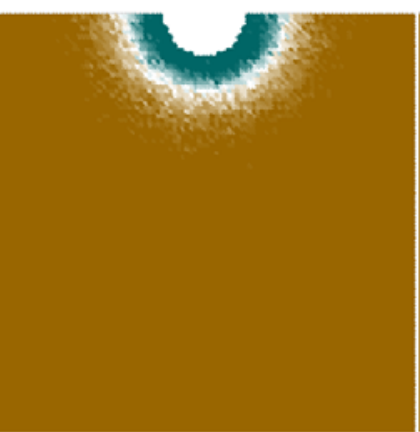

$\mathrm{C}$

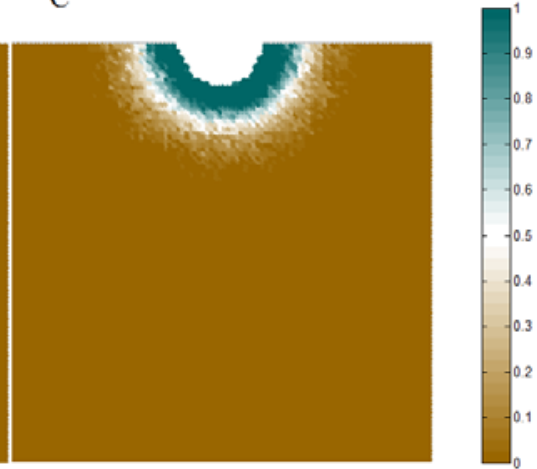

Fig. 7 Time step size convergence analysis for the damage index map at time $=20 \mathrm{~s}$ and $\eta=$ $0.2 \mathrm{~V}: \mathrm{dt}=5 \mathrm{~s}(\mathrm{~A}), \mathrm{dt}=0.5 \mathrm{~s}(\mathrm{~B}), \mathrm{dt}=0.05 \mathrm{~s}(\mathrm{C})$
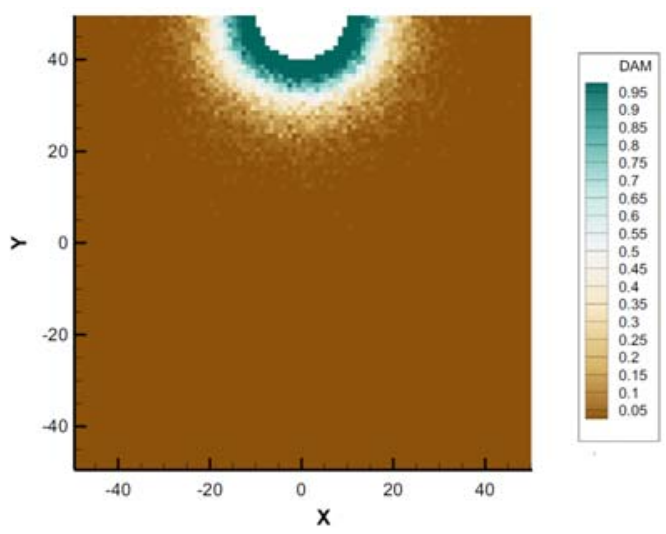
Fig. 8 Damage index map at time $=20 \mathrm{~s}$ for the case with $\eta=0.2 \mathrm{~V}$ reported in Chen and

Bobaru (2015)

In Figs. 7 and 8, only the particles in solid phase are shown. The liquid/solid interface does not advance within the first $20 \mathrm{~s}$, but a layer constituted by solid particles with damage index value equals to one is formed at the interface. As explained earlier, if a generic particle has a damage index value equals to one, it means that all of its bonds have been broken. Thus, in this case, the particle is totally disconnected from the rest of the body and the solution can penetrate underneath. In order to mimic this phenomenon, the approach used in Chen and Bobaru (2015) is adopted. According to this approach, the phase of the node is changed from solid to liquid when $d=1$ even if $C>C_{s a t}$. As shown in Fig. 9, the results produced by the present model are in quantitative agreement with the study reported in Chen and Bobaru (2015).
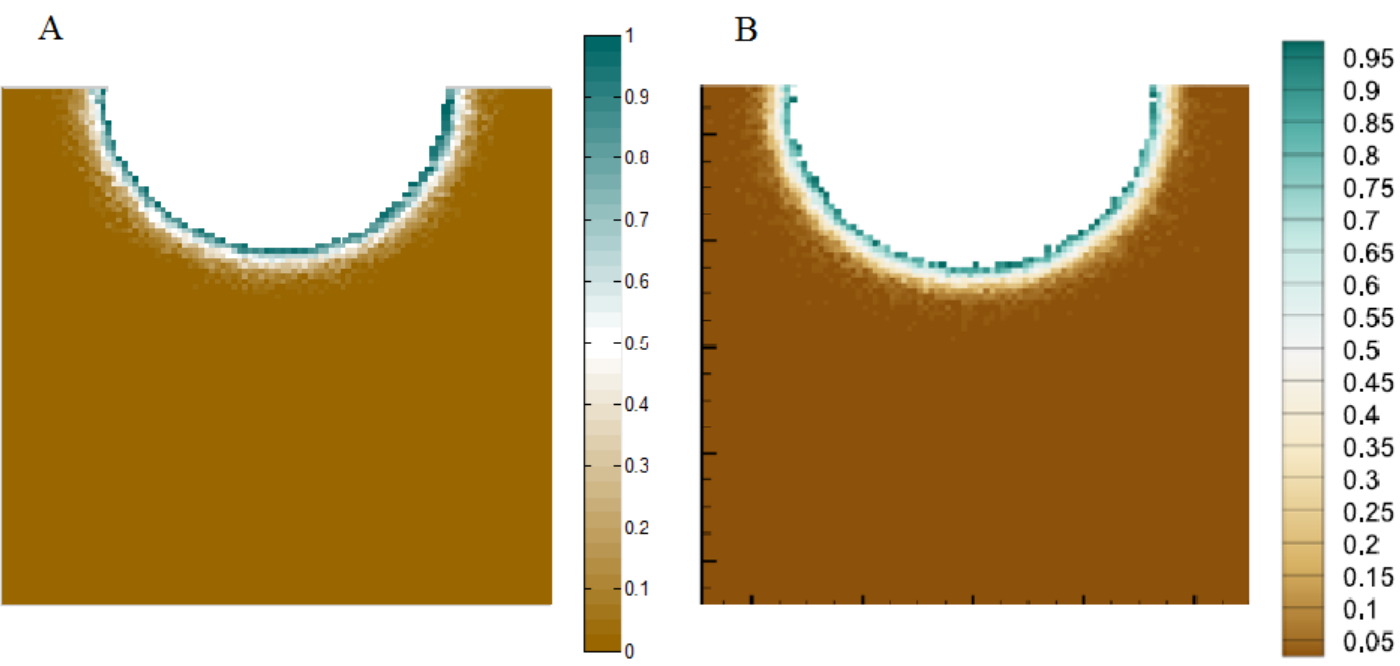

Fig. 9 Damage index map at time $=20 \mathrm{~s}$ for the case with $\eta=0.2 \mathrm{~V}$ : present numerical model (A) and other numerical model (Chen and Bobaru, 2015) (B)

\subsection{Realistic pit morphologies}

In reality, corrosion pits can have complex geometries, which are often very different from the simple case considered in the previous section. The PD model of pitting corrosion can be extended to model realistic pit morphologies. There may be 
several different approaches that can be used in this regard. The procedure adopted in this study, which is used during the preparation of the model, consists of the following two steps:

1. Selection of the region of the domain where the pit is expected to propagate.

2. For all the nodes that do not belong to this region, an artificial effective coefficient of diffusion in the metal $D_{s d}^{*}$ is used. The ratio between $D_{s d}^{*}$ and $D_{s d}$ is always smaller than 1 and is called "coefficient of pit morphology" $c_{p m}:$

$$
c_{p m}=\frac{D_{s d}^{*}}{D_{s d}}<1
$$

The following boundary condition is applied to the blue region of length $6 \mu \mathrm{m}$ in Fig. 10 as $C(x, t)=0$.

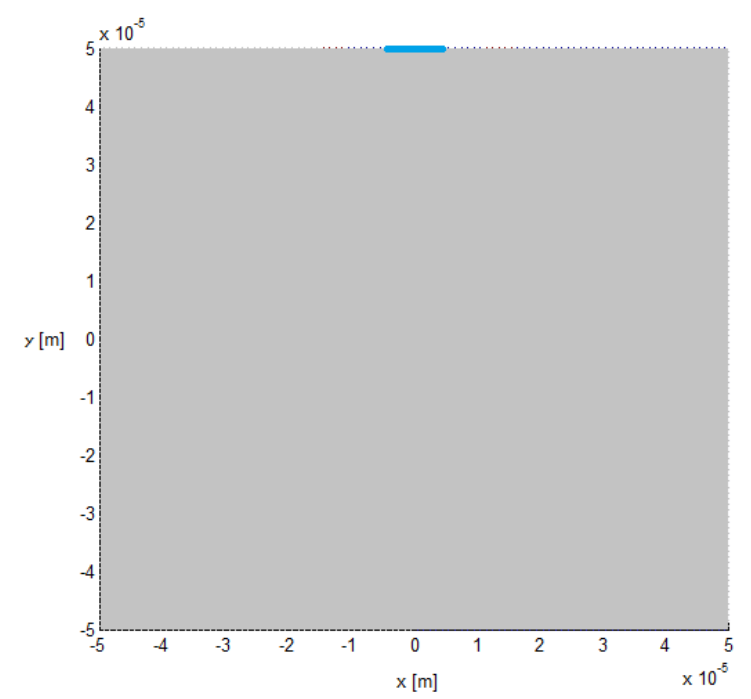

Fig. 10 PD model for the prediction of realistic pit morphologies: metal (grey colour) and initial liquid/solid interface (blue colour)

As shown in Fig. 11, if the morphology of the pit is known a priori, then the PD model is able to reproduce the expected shape of the pit cavity. The material 
properties and numerical parameters used for the simulations in Fig. 11 are the same as those used in previous section. The only difference is the introduction of $c_{p m}$, which is assumed to be $1 \%$.

A

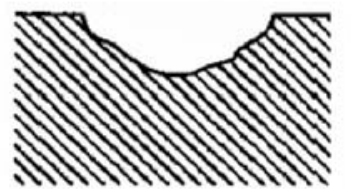

Wide and shallow

B

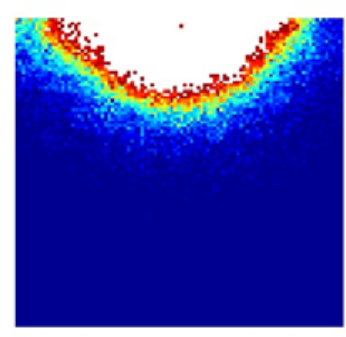

time $=500 \mathrm{~s}$

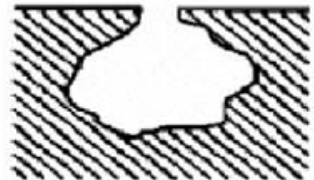

Subsurface

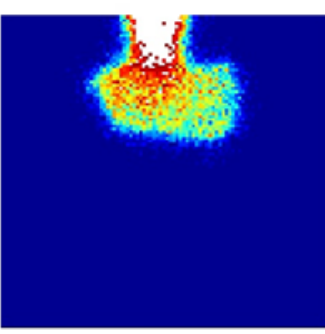

time $=350 \mathrm{~s}$

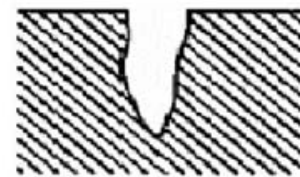

Narrow and deep

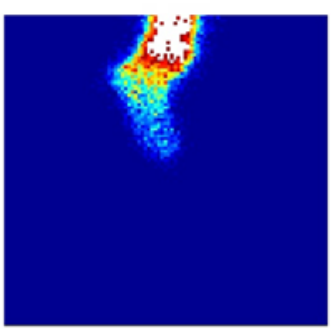

time $=150 \mathrm{~s}$

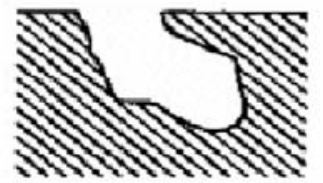

Undercutting

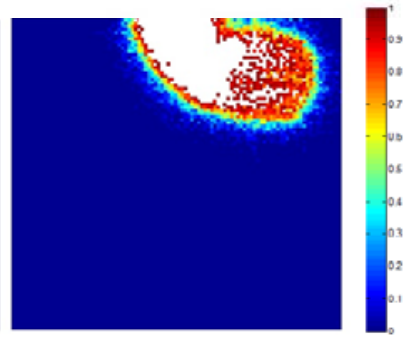

time $=600 \mathrm{~s}$

Fig. 11 Realistic pit morphologies (Hoeppner, 2011) (A), damage index map obtained with the present numerical model (B)

\subsection{Pitting corrosion in a cluster of cathodic intermetallic particles}

It is well-known that the metallurgical characteristics of the material can affect the evolution of corrosion pits. In this analysis, pitting propagation in a cluster of cathodic intermetallic particles (IMPs) is investigated. The material properties and numerical parameters are the same as those used in the previous section. IMPs are created through node selection during the generation of the model and they are represented by the application of the following boundary condition to all those nodes that belong to the IMPs domain as $C(x, t)=C_{\text {solid }}$. 


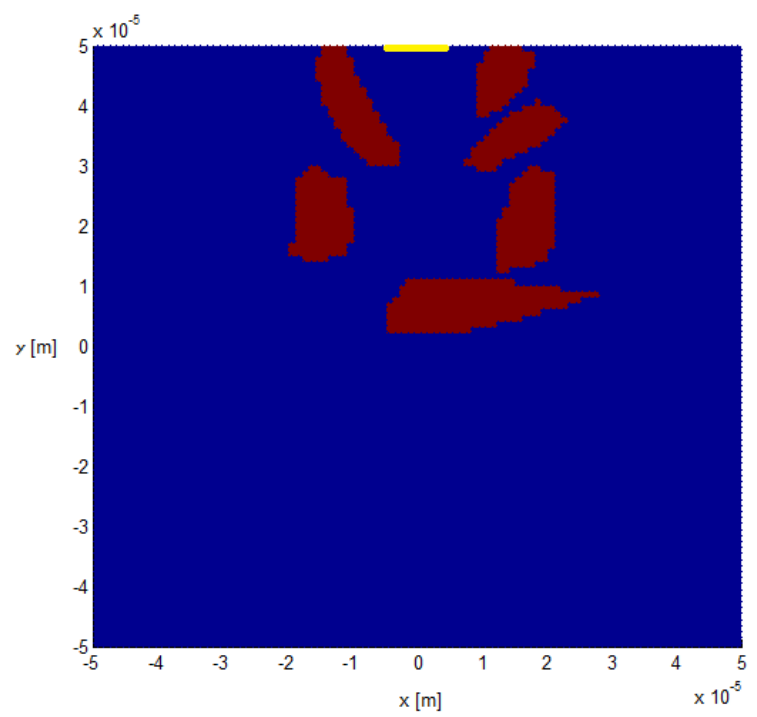

Fig. 12 PD model of pitting corrosion in a cluster of IMPs: metal (blue colour), corrosive solution (yellow colour) and IMPs (red colour)

As for the analyses described in previous sections, the following boundary condition is applied to the yellow region of length $6 \mu \mathrm{m}$ in Fig. 12 as $C(x, t)=0$. As shown in Fig. 13, pit damage is channelled by the cluster of IMPs and the lower part of the plate is partially shielded by the IMP at the bottom of the cluster. In order to better visualise the pit cavity, only solid nodes are shown in Fig. 13.

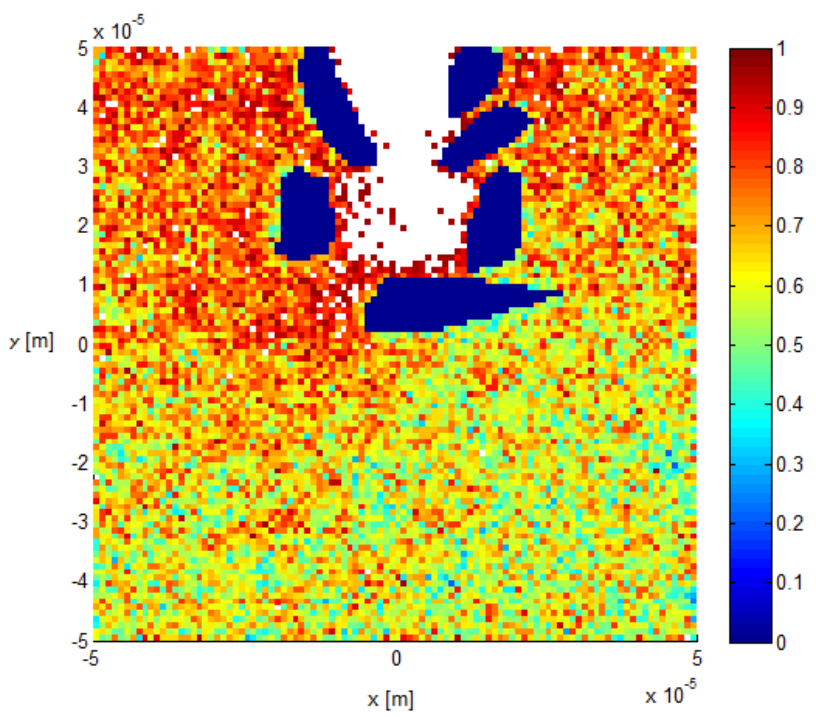

Fig. 13 Pit evolution in a cluster of IMPs. Damage index map at time $=2500$ s: no dissolution (blue colour) and close to dissolution (red colour) 


\section{Conclusions}

The ultimate goal of this study was to use peridynamics to produce a nonconventional and more effective numerical framework that can be helpful in failure analysis and design of new corrosion-resistant materials. For this purpose, a new numerical model based on peridynamics to predict pitting corrosion damage was developed by extending an existing model to allow for 1) the reproduction of realistic pitting morphologies, 2) modelling of microstructural effects such as the presence of intermetallic particles and 3) the reduction of the computational cost of the simulations. Three different cases were considered to validate the developed model and demonstrate its capabilities. In the first case, pitting corrosion evolution in a stainless steel plate was simulated and a suitable time step size was determined after performing a convergence analysis. In the second case, realistic pit morphologies were obtained by using the current framework. Finally, the effect of intermetallic particles on pitting corrosion evolution was investigated and it was found that the intermetallic particles have a significant effect on pitting corrosion damage evolution.

\section{Acknowledgement}

The authors would like to acknowledge Prof. Ivan Cole, Dr. Dayalan Gunasegaram and Dr. Fiona Chen from CSIRO Laboratories in Clayton, Australia for useful discussions and suggestions. 


\section{References}

Amani, J., Oterkus, E., Areias, P. M., Zi, G., Nguyen-Thoi, T. and Rabczuk, T., 2016, "A non-ordinary state-based peridynamics formulation for thermoplastic fracture," International Journal of Impact Engineering, Vol. 87, pp. 83-94.

Bhandari, J., Khan, F., Abbassi, R., Garaniya, V. and Ojeda, R., 2015. Modelling of pitting corrosion in marine and offshore steel structures - A technical review. Journal of Loss Prevention in the Process Industries, 37, pp.39-62.

Chen, Z. and Bobaru, F., 2015. Peridynamic modeling of pitting corrosion damage. Journal of the Mechanics and Physics of Solids, 78, pp.352-381.

Chen, Z., Zhang, G. \& Bobaru, F., 2015. The Influence of Passive Film Damage on Pitting Corrosion. Journal of the Electrochemical Society, 163(2), pp.C19-C24.

De Meo, D., Diyaroglu, C., Zhu, N., Oterkus, E. and Siddiq, M. A., 2016. Modelling of stress-corrosion cracking by using peridynamics. International Journal of Hydrogen Energy, 41, pp.6593-6609.

Duddu, R., 2014. Numerical modeling of corrosion pit propagation using the combined extended finite element and level set method. Computational Mechanics, 54, pp.613-627.

Duddu, R., Kota, N. and Qidwai, S., 2015. An extended finite element model of crevice and pitting corrosion. In IMECE2015-50423. pp. 1-11.

Gavrilov, S., Vankeerberghen, M., Nelissen, G. and Deconinck, J., 2007. Finite element calculation of crack propagation in type 304 stainless steel in diluted sulphuric acid solutions. Corrosion Science, 49, pp.980-999. 
Gerstle, W., Silling, S., Read, D., Tewary, V. and Lehoucq, R., 2008. Peridynamic simulation of electromigration. Computers, Materials and Continua, 8(2), pp. 75-92.

Hoeppner, D. W., 2011. Pitting Corrosion: Morphology and Characterization. RtoAg-Avt-140, pp.5.1-5.16.

Isaacs, H. S., Cho, J. H., Rivers, M. L. and Sutton, S. R., 1995. In Situ X-Ray Microprobe Study of Salt Layers during Anodic Dissolution of Stainless Steel in Chloride Solution. Journal of The Electrochemical Society, 142(4), pp.11111118 .

Kuo, H. C. and Landolt, D., 1975. Rotating disc electrode study of anodic dissolution or iron in concentrated chloride media. Electrochimica Acta, 20(May), pp.393-399.

Laycock, N. J. and White, S. P., 2001. Computer simulation of single pit propagation in stainless steel under potentiostatic control. J. Electrochem. Soc., 148, pp.B264-B275.

Madenci, E. and Oterkus, E., 2014. Peridynamic theory and its applications. Springer.

Onishi, Y., Takiyasu, J., Amaya, K., Yakuwa, H. and Hayabusa, K., 2012. Numerical method for time-dependent localized corrosion analysis with moving boundaries by combining the finite volume method and voxel method. Corrosion Science, 63, pp.210-224.

Oterkus, E. and Madenci, E., 2012, "Peridynamics for Failure Prediction in Composites," $53^{\text {th }}$ AIAA/ASME/ASCE/AHS/ASC Structures, Structural Dynamics, and Materials Conference, Honolulu, HI, Paper No.2012-1692. 
Oterkus, S., Fox, J. and Madenci, E., 2013, "Simulation of electro-migration through peridynamics," Electronic Components and Technology Conference (ECTC), 2013 IEEE 63rd (pp. 1488-1493). IEEE.

Oterkus, S. and Madenci, E., 2014, "Fully coupled thermomechanical analysis of fiber reinforced composites using peridynamics," 55th AIAA/ASME/ASCE/AHS/SC Structures, Structural Dynamics, and Materials Conference-SciTech Forum and Exposition 2014.

Oterkus, S., Madenci, E., Oterkus, E., Hwang Y., Bae, J. and Han, S., 2014, "HygroThermo-Mechanical Analysis and Failure Prediction in Electronic Packages by Using Peridynamics," 64th Electronic Components \& Technology Conference, Lake Buena Vista, Florida, USA.

Scheiner, S., Hellmich, C., 2009. Finite Volume model for diffusion- and activationcontrolled pitting corrosion of stainless steel. Computer Methods in Applied Mechanics and Engineering, 198(37-40), pp.2898-2910.

Sharland, S. M., Jackson, C.P. and Diver, A.J., 1989. A finite-element model of the propagation of corrosion crevices and pits. Corrosion Science, 29(9), pp.11491166.

Silling, S. A., 2000. Reformulation of elasticity theory for discontinuities and longrange forces. Journal of the Mechanics and Physics of Solids, 48, pp.175-209.

Song, W., Martin, H. J., Hicks, A., Seely, D., Walton, C. A., Lawrimore, W. B., Wang, P. T. and Horstemeyer, M. F., 2014. Corrosion behaviour of extruded AM30 magnesium alloy under salt-spray and immersion environments. Corrosion Science, 78, pp.353-368.

Vagbharathi, A. S. and Gopalakrishnan, S., 2014. An extended finite-element model coupled with level set method for analysis of growth of corrosion pits in 
metallic structures. In Proceedings of the Royal Society A: Mathematical, Physical and Engineering Sciences.

Van der Weeën, P., Zimer, A. M., Pereira, E. C., Mascaro, L. H., Bruno, O. M. and De Baets, B., 2014. Modeling pitting corrosion by means of a 3D discrete stochastic model. Corrosion Science, 82, pp.133-144.

Walton, J. C., 1990. Mathematical modeling of mass transport and chemical reaction in crevice and pitting corrosion. Corrosion Science, 30(8-9), pp.915-928.

Xiao, J. and Chaudhuri, S., 2011. Predictive modeling of localized corrosion: An application to aluminum alloys. Electrochimica Acta, 56(16), pp.5630-5641. 